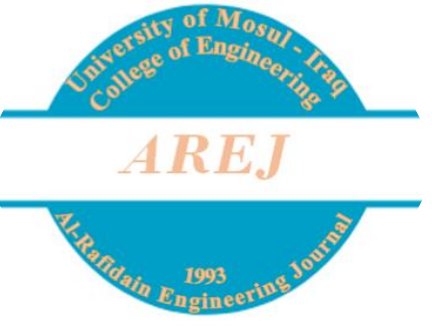

Al-Rafidain Engineering Journal (AREJ)

Vol.26, No.2, October 2021, pp.259-266

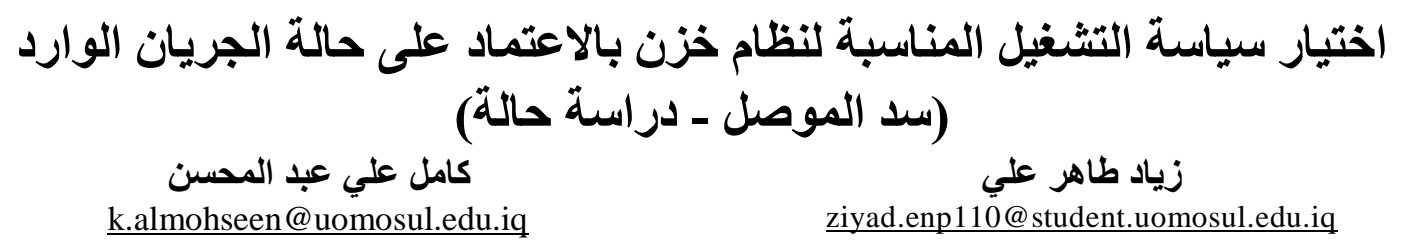

جامعة الموصل - كلية الهندسة ـ قسم هندسة السدود و الموارد المائية

تاريخ القبول: 12/8/2021

تاريخ الاستلام: 17/6/2021

| (لمستخلص

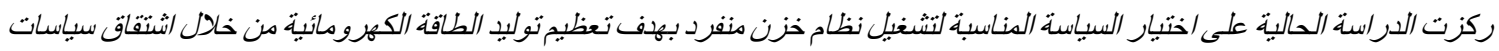

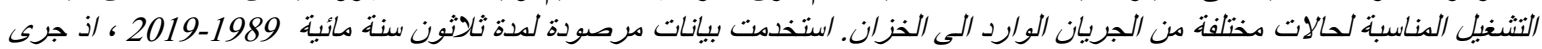

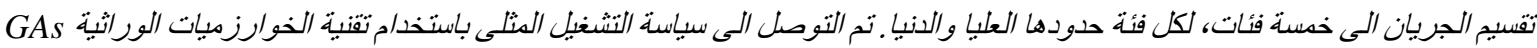

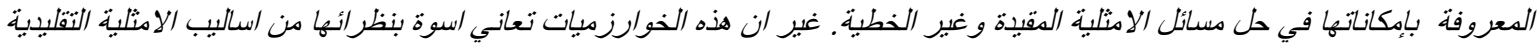

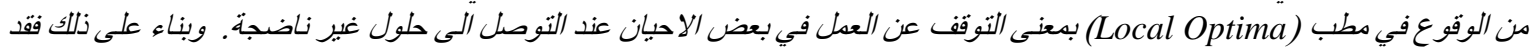

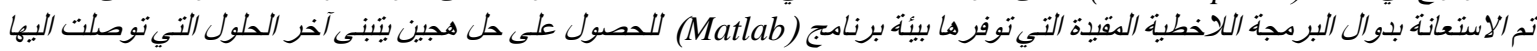

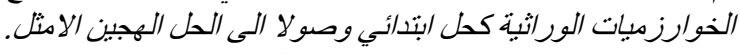

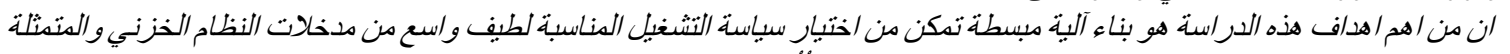

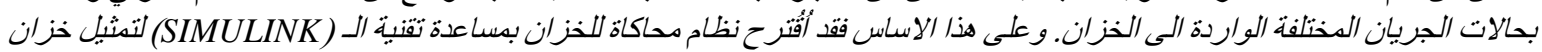

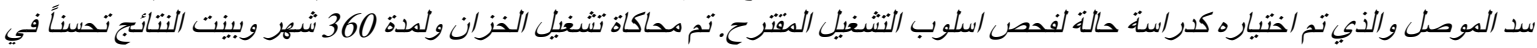

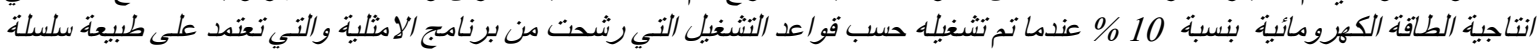
الجريان الواردة للخزان على مديى 30 عاماً.

الكلمات الدالة:

سياسة التشغيل ، الامثلية ، الخوارزمبات الوراثية ، الدحاكاة.

This is an open access article under the CC BY 4.0 license (http://creativecommons.org/licenses/by/4.0/). https://rengj.mosuljournals.com

1

أنظمة الخزن لا يتم تشغيلها و إدارتها بكفاءة بالاعتماد على سياسات

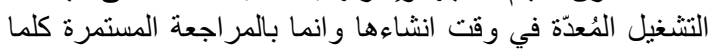

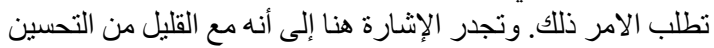

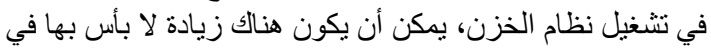

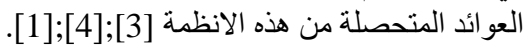

تم دراسة سياسات تشغيل الخزانات المائية بشكل مستفيض على الخي

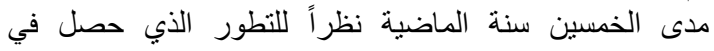

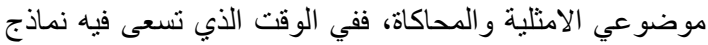

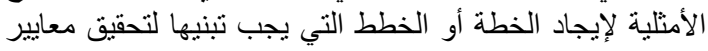

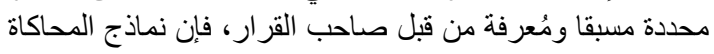

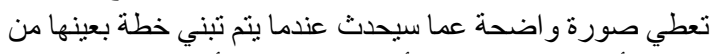

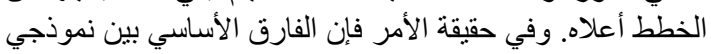

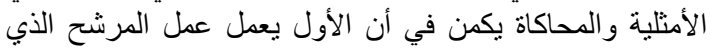

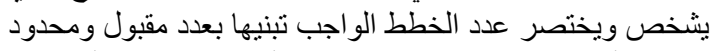

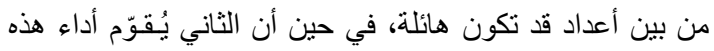

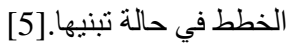

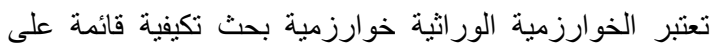

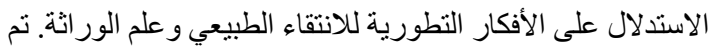

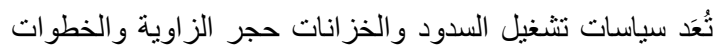

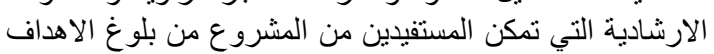

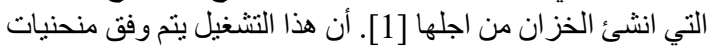
تسمى Rule curves و التي تعطي فكرة واضحة عن التهي كمية المياه

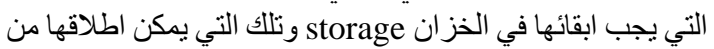

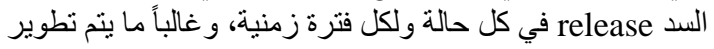

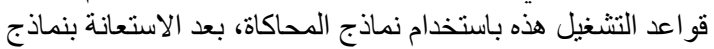

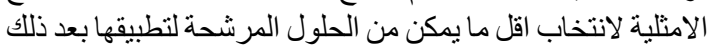

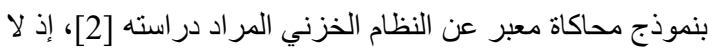

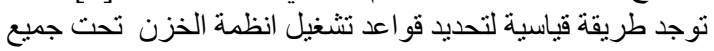
الظروف التي يمر بها الخزان.

تم استخدام العديد من طرق الأمثلية ونماذج المحاكاة في تحديد

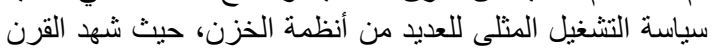

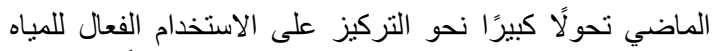

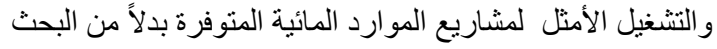

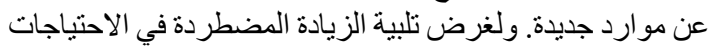

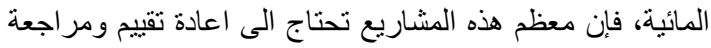

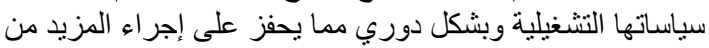

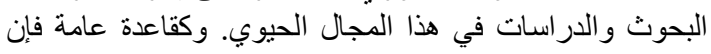


يتبين مما ورد اعلاه عدم وجود آلية معينة لاختيار سياسة تشغيل

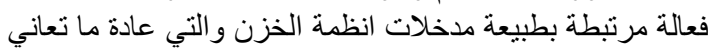

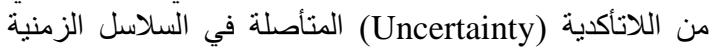

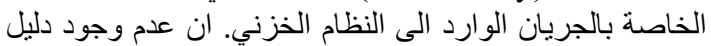

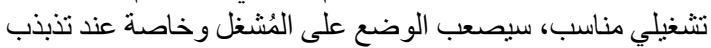

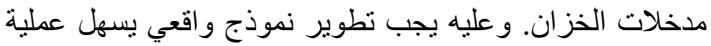

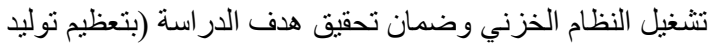

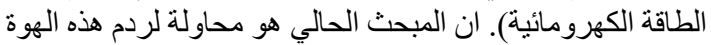
و استنباط الآلية المطلوبة.

\section{بناء نموذج الامثلية (Optimization Model)}

تتمثل دالة الهدف في هذه الدر اسة بتعظيم توليد القدرة الكهرومائية طبقاً لقيود خطية و غير خطية متعددة، يمكن التعبير عنه لهنها رياضيًا:

$$
\begin{aligned}
& \operatorname{Max} \operatorname{MOP}(\mathrm{x}) \ldots . . . . . . .(1) \\
& M O P=\sum_{t=1}^{12} \eta \times \mathrm{R}_{\mathrm{t}} \times\left(H_{t}\right)
\end{aligned}
$$

Subject to :

$$
\begin{aligned}
& \mathrm{S}_{\min } \leq \mathrm{S}_{\mathrm{t}} \leq \mathrm{S}_{\max } \\
& \mathrm{R}_{\mathrm{t}} \geq \mathrm{D}_{\mathrm{t}} \\
& \mathrm{R}_{\mathrm{t}} \leq \mathrm{R}_{\max } \\
& \sum_{1}^{12} \mathrm{R}_{\mathrm{t}} \leq \sum_{1}^{12} \mathrm{I}_{t} \\
& S_{t}=S_{t-1}+I_{t}-R_{t}+P_{t}-L_{t}
\end{aligned}
$$

تصميم المفهوم الأساسي لـ GAs لمحاكاة العمليات التطورية في

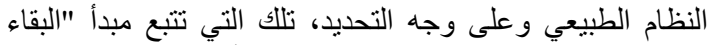

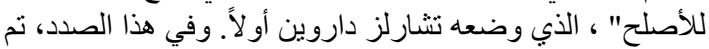

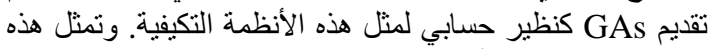

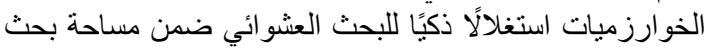

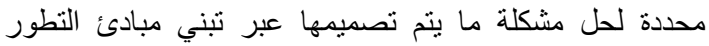

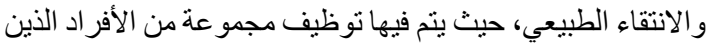

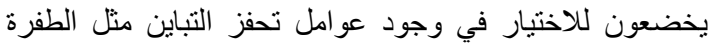

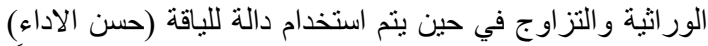

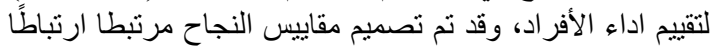

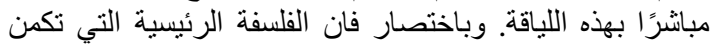

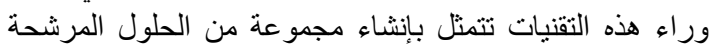

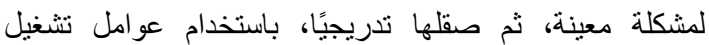

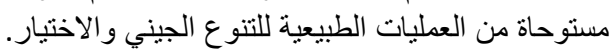

قدّم [6] مر اجعة عن تطبيقات الخوارزميات التطورية EA لتشغيل

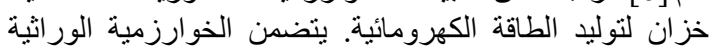
GAs ، و التطور التفاضلي، وخلص إلى أن GAs

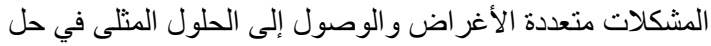

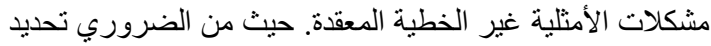

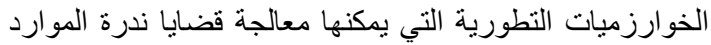
المائية وتأثير ها على التنمية الاقتصـادية.

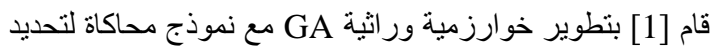

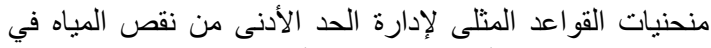

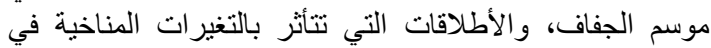

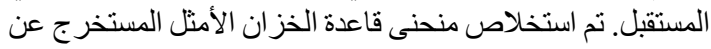

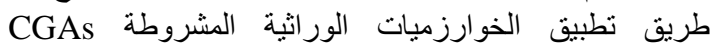

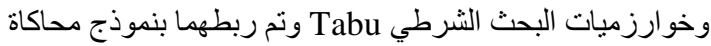

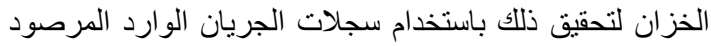
والجريان المستقبلي المتوقع، وذلك لمعالجة حالة نقص الدان المياه و الإطلاقات الز ائدة .

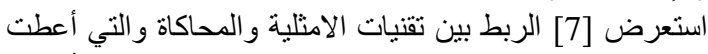

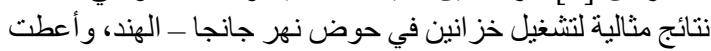

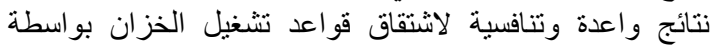

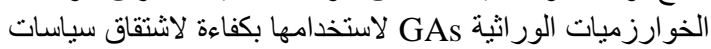

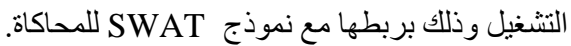

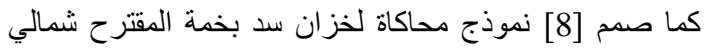

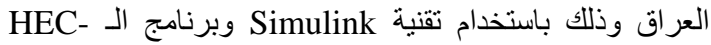

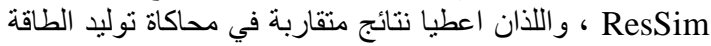

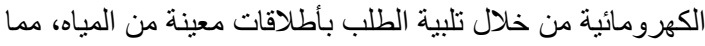

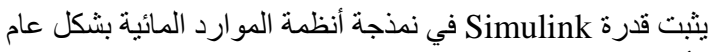
و أنظمة الخز انات بشكل خاص.

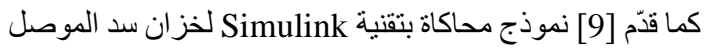

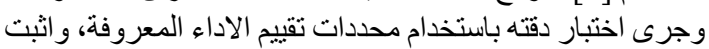
ان النموذج و اعداً ومرناً في التطبيقات الو اقعية.

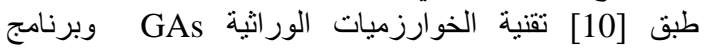
Optimization Modeling Software LINGO والتشغيل الأمثل لخز ان متعدد الأغر اض وحقق القيم الأكثر موثوقية

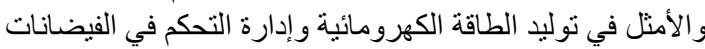

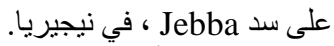

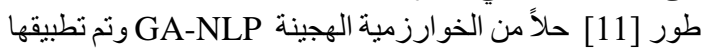
على نظام خزان Nagarjuna sagar في الهند، لاشتقاق منحنيات

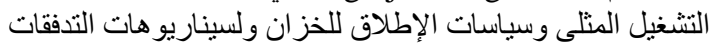

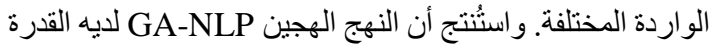
على الأداء بكفاءة، إذا ما تم تطبيقه في إيجاد السياسات الني التشغيلية

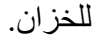


الجريان الشهري واعلى جريان مسجل (Upper quartile)، ولفئل

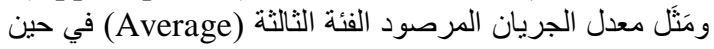

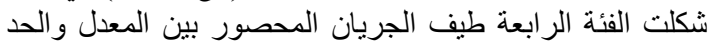

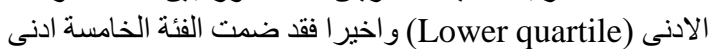

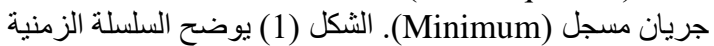

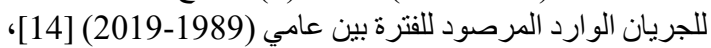
أما فئات الجريان الخمسة المقترحة مقاسة بـ(MCM/year) فكانت كالاتي : فات الجريان

• فئة الجريان بين 6000-10000 لاقل جريان سنوي وارد و الذي سيخضع للسياسة التشغيلية (1000 (Min. Policy).

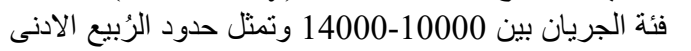

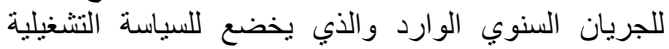
(Lower Quartile Policy) • أما الجريان بين 14000-18000 فمثلت حدود فئة معدل الجريان السنوي الوارد والذي يخضع للسيانة النتئية (Average Policy) • الجريان بين 18000-25000 فمثل ححود فئة الجريان للربيع

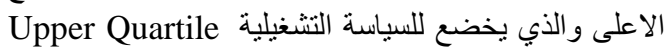
Policy • فيما مثلت السياسة التشغيلية القصوى Max Policy فئة حدود

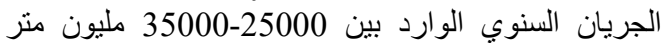
مكعب سنوياً. اجريت عملية تشغيل نموذج الامثلية الواردة تفاصليه اعلاه بتبني

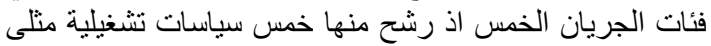

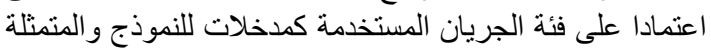

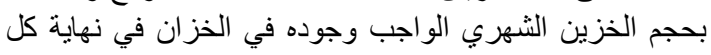

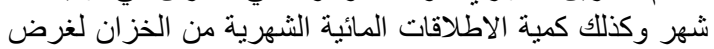

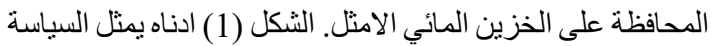

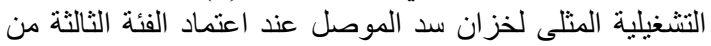
تقسيمات الجريان الوارد الى خزان الهي سد الموصل و المتمثلة بمعدل الجريان لفترة (30) عاماً.

جرى بنفس الطريقة الحصول على السياسات النشغيلية الاربع

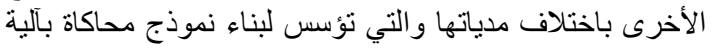

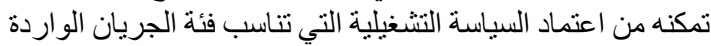

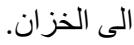

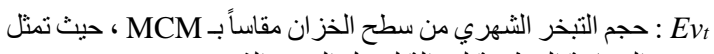
المساحة السطحية له دالة لمعدل الخزن الثزن الثهري.

seepage من خزان سد الموصل مقاسة بالثهر

علماً ان قيم المعدلات الثهرية لعمق التبخر من الخزان و الامطار الساقطة عليه موضحة في الجدول (1) [13].

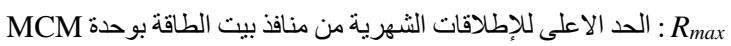

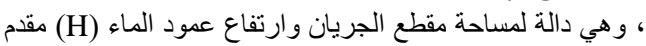

السد الذي هو دالة الخزن، و يعبر عن هذه الاطلاقات بالعلاقة :

$$
\boldsymbol{R}_{\text {max }}=\mathrm{C}_{\mathrm{d}} \mathrm{A} \sqrt{2 \mathrm{~g} \mathrm{H}}
$$

حيث : معامل التصريف عند المبثق (Discharge coefficient) هو البه النسبة بين التصريف الحقيقي إلي التصريف النظري. ونم استنتاجه حسابيا بقيمة (0.6). مساحة مقطع الجريان (m²) لوحدات التوليد الاربعة في حالة التشغيل : A الكلي لجميع الوحدات.

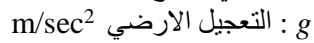

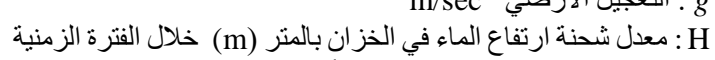

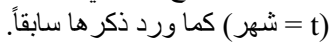

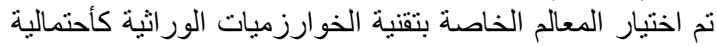

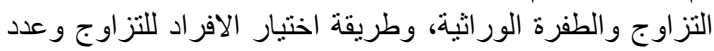

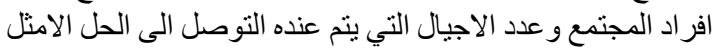

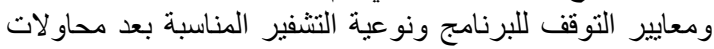
عديدة وقد كانت كالآتي: ائر حجم المجتمع هو 300 ؛ أحتمالية التزاوج اون او التقاطع فقد تم تعيينها

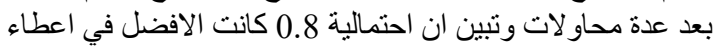

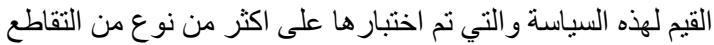

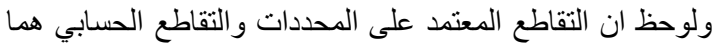

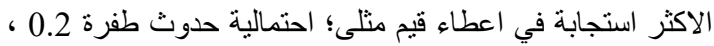

و وعدد الأجيال هو 2500. ولغرض تهيئة مدخلات النموذج المتمثلة بالجريان الثهري الوارد

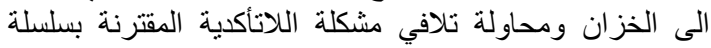

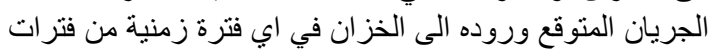

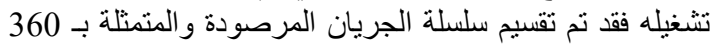

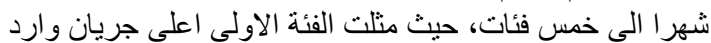
(Maximum)

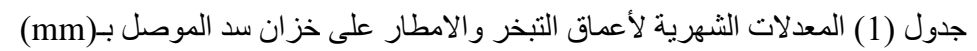

\begin{tabular}{|c|c|c|c|c|c|c|c|c|c|c|c|c|}
\hline ايلول & اب & تموز & حزير ان & ايار & نيسان & اذار & شباط & كانون2 & كانون1 & تشرين2 & تشرين1 & الثهر \\
\hline 218 & 340 & 408 & 340 & 213 & 116 & 70 & 40 & 30 & 30 & 52 & 138 & تبخر \\
\hline 0 & 0 & 0 & 0 & 23.8 & 54.4 & 70 & 65 & 68.1 & 63.4 & 36.8 & 8.7 & امطار \\
\hline
\end{tabular}

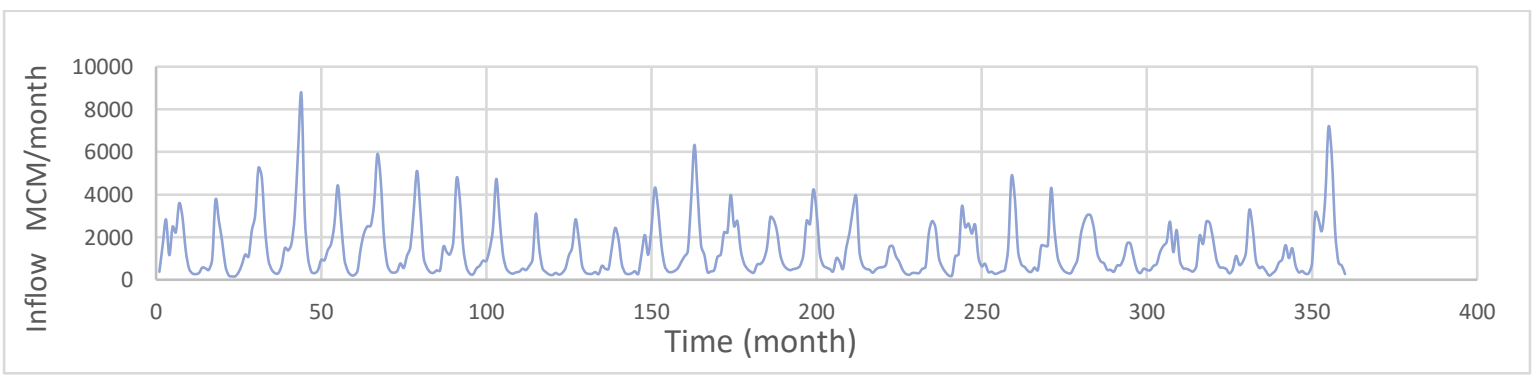

الثكل (1) السلسلة الزمنية الثهرية للجريان الوارد المرصود الى خزان سد الموصل 


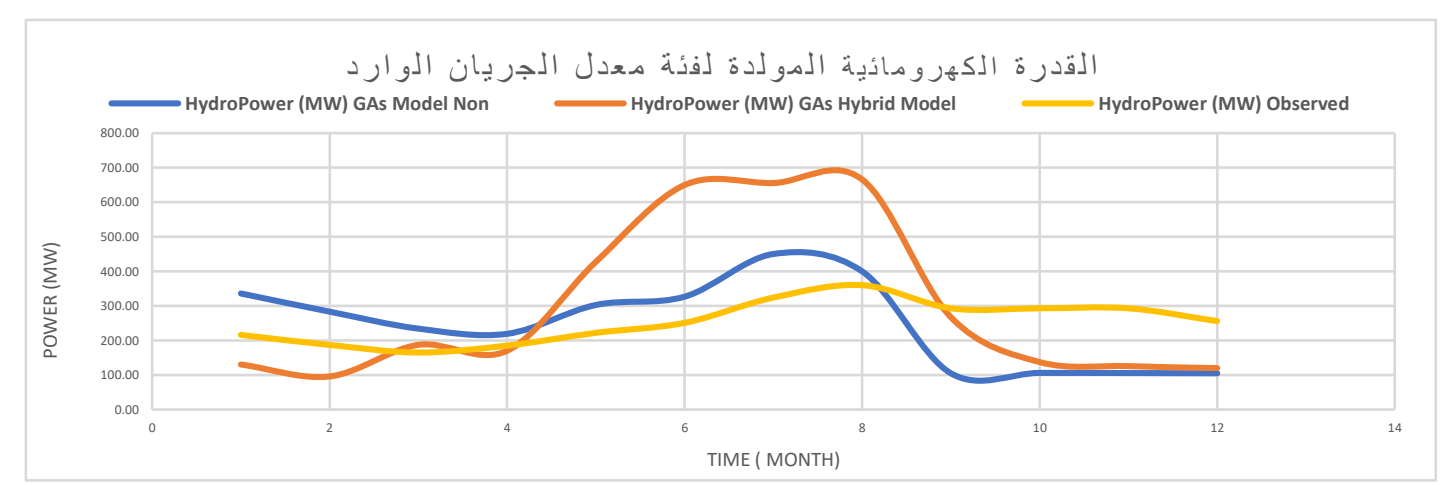

Average Policy الثكل (2) السياسة التشغيلية المثلى لخزان سد الموصل باعتماد معدل الجريان الوارد

للمرونة العالية التي تتمتع بها هذه التقنبة وامكاناتها في الاستفادة

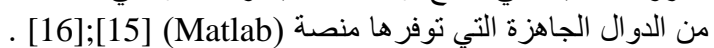

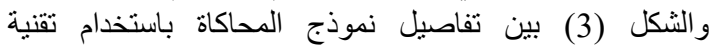
بينما يبين الثكل (4) بئن (Simulink)

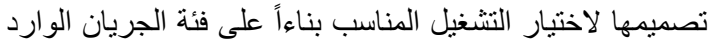
ويعتمد على كتل العمليات المنطقية والثرطية (If statement) في عملية الاختيار :
نموذج المحاكاة (Simulation Model)

تتطلب الآلية المقترحة في هذا المبحث بان يتم اختيار السياسة

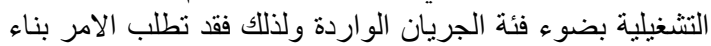

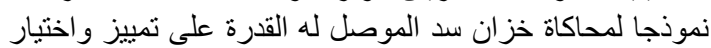

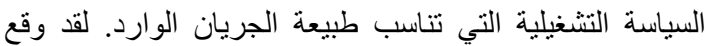

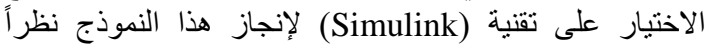

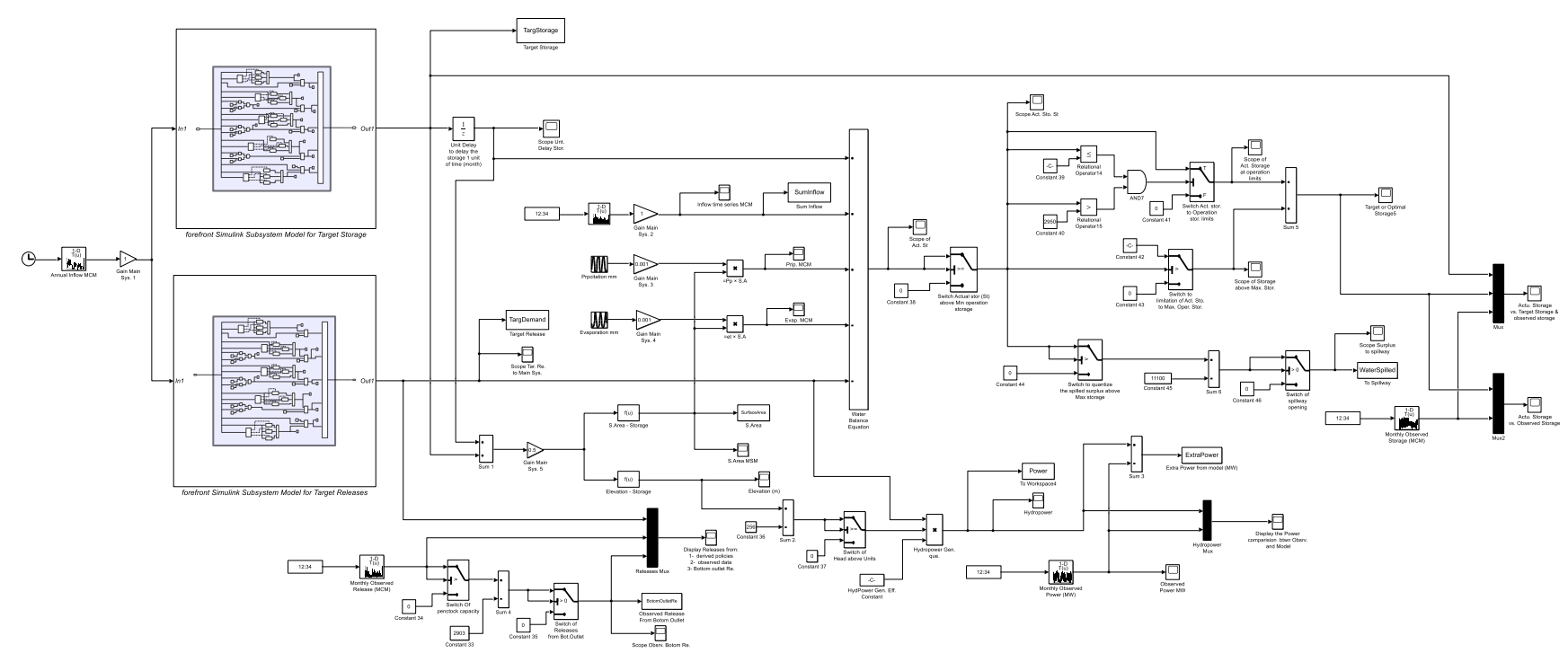

الشكل (3) نموذج المحاكاة المقترح باستخدام تقتية (Simulink) 


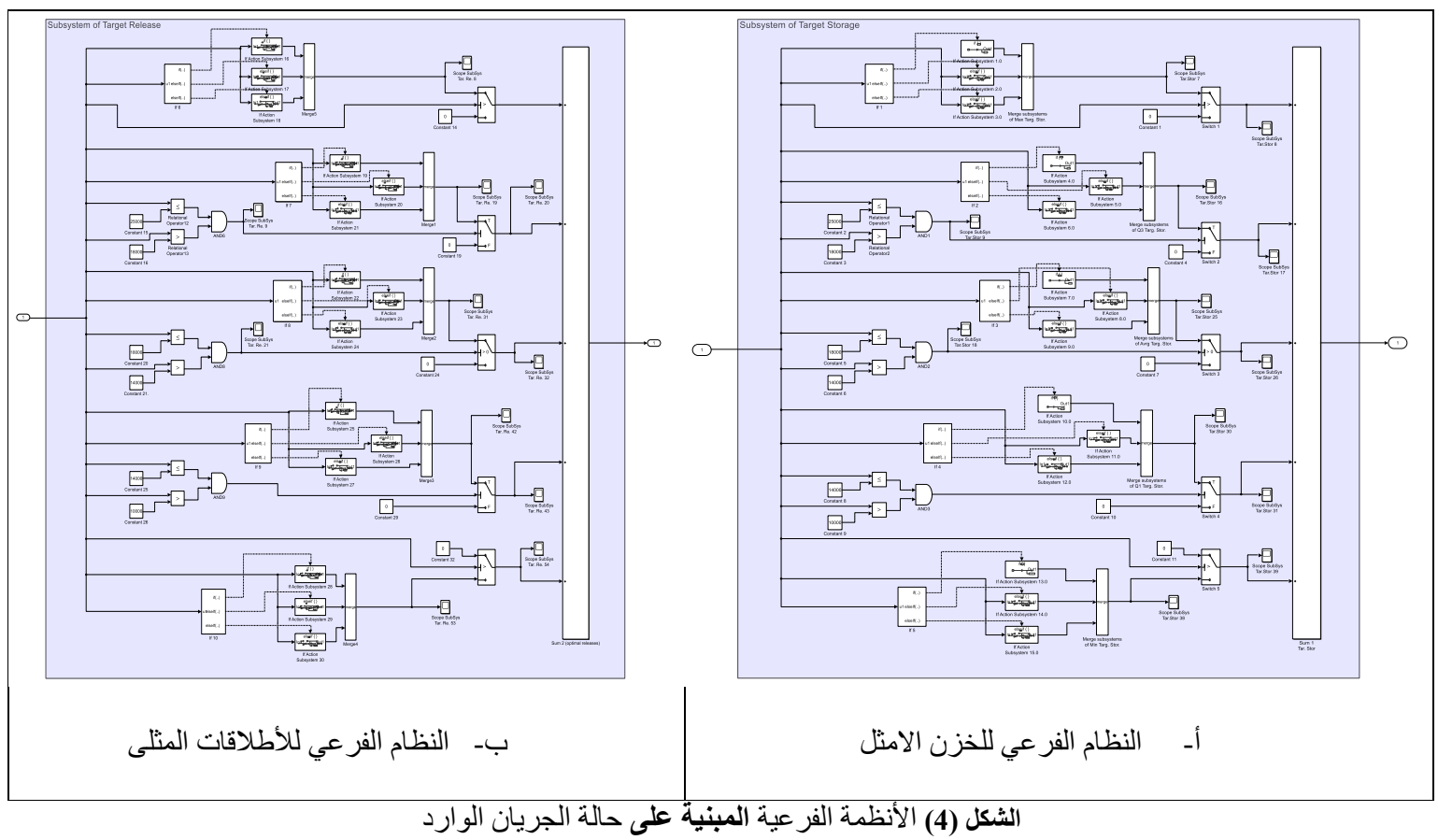

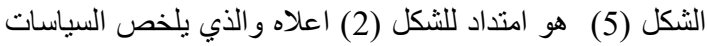

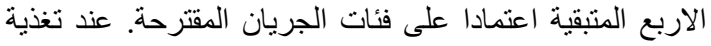

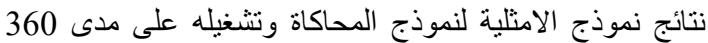

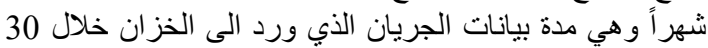

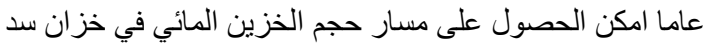

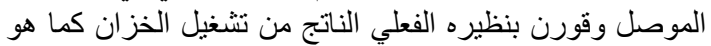

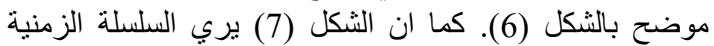

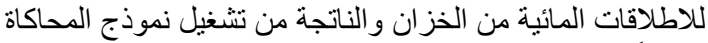

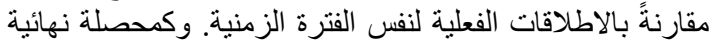

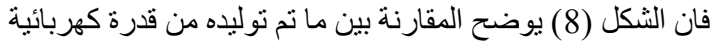

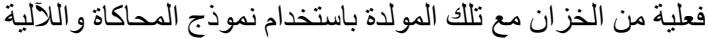
المعتمدة في هذا المبحث لنفس الفترة الزمنية. دللت نتائج نموذج الامثلية على امكانية التوصل الى السياسة

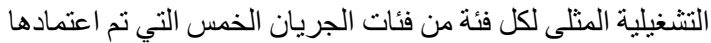

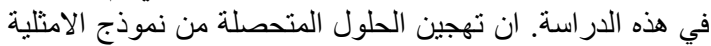

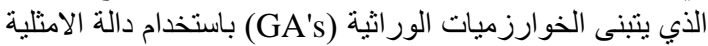
الجاهزة (fmincon) في منصة الماتلاب والتي التي تعالج اللاخطية

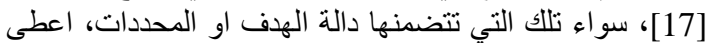

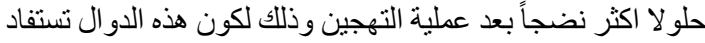

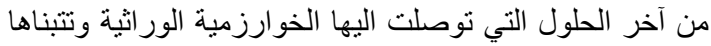

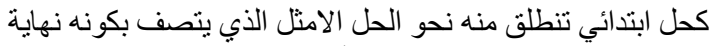
عظمى (Global Optima) بدلاً من الحل الابتدائي الذي رئل

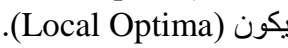

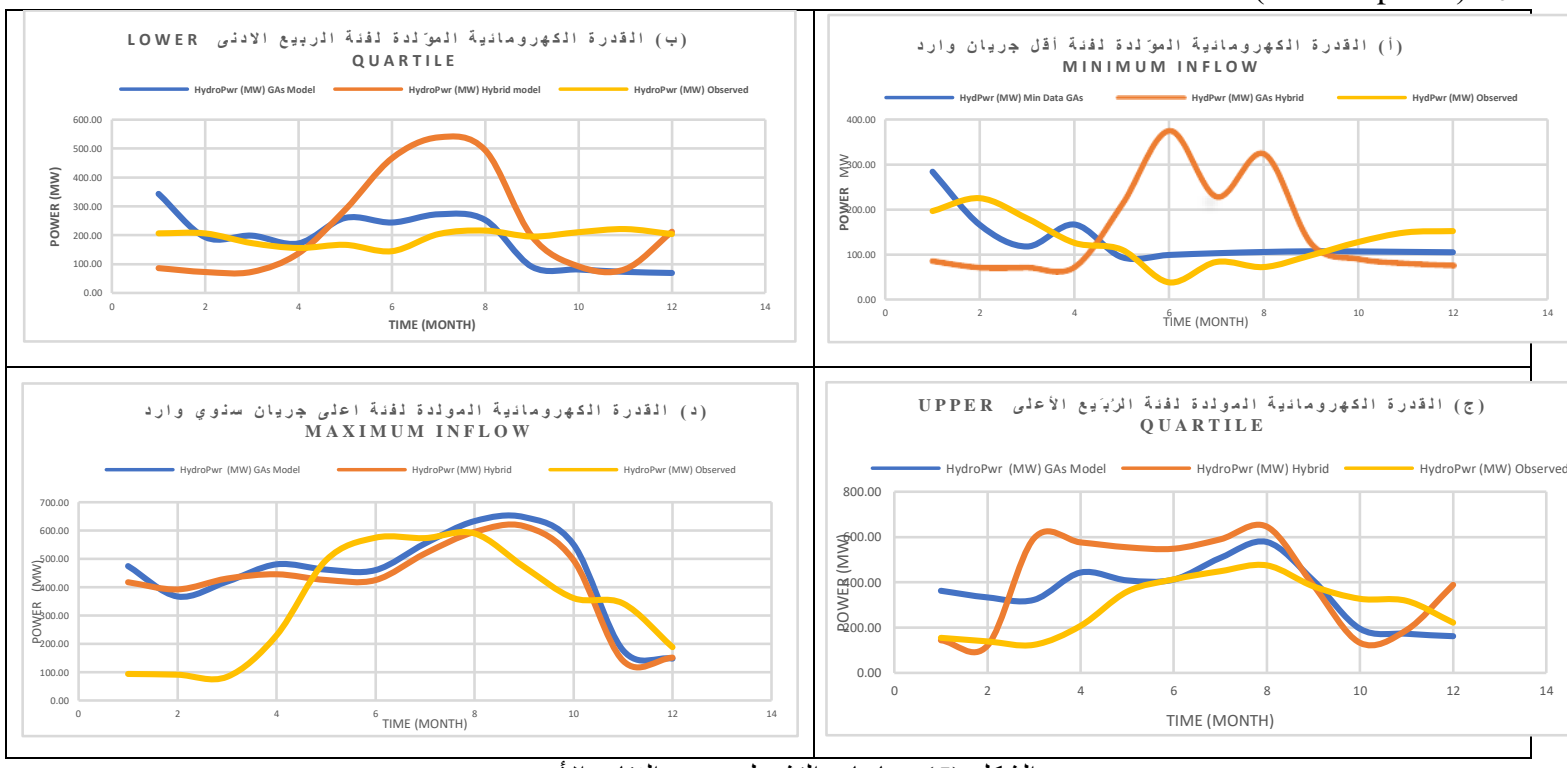

الثكل (5) سياسات التشغيل حسب الفئات الأخرى 


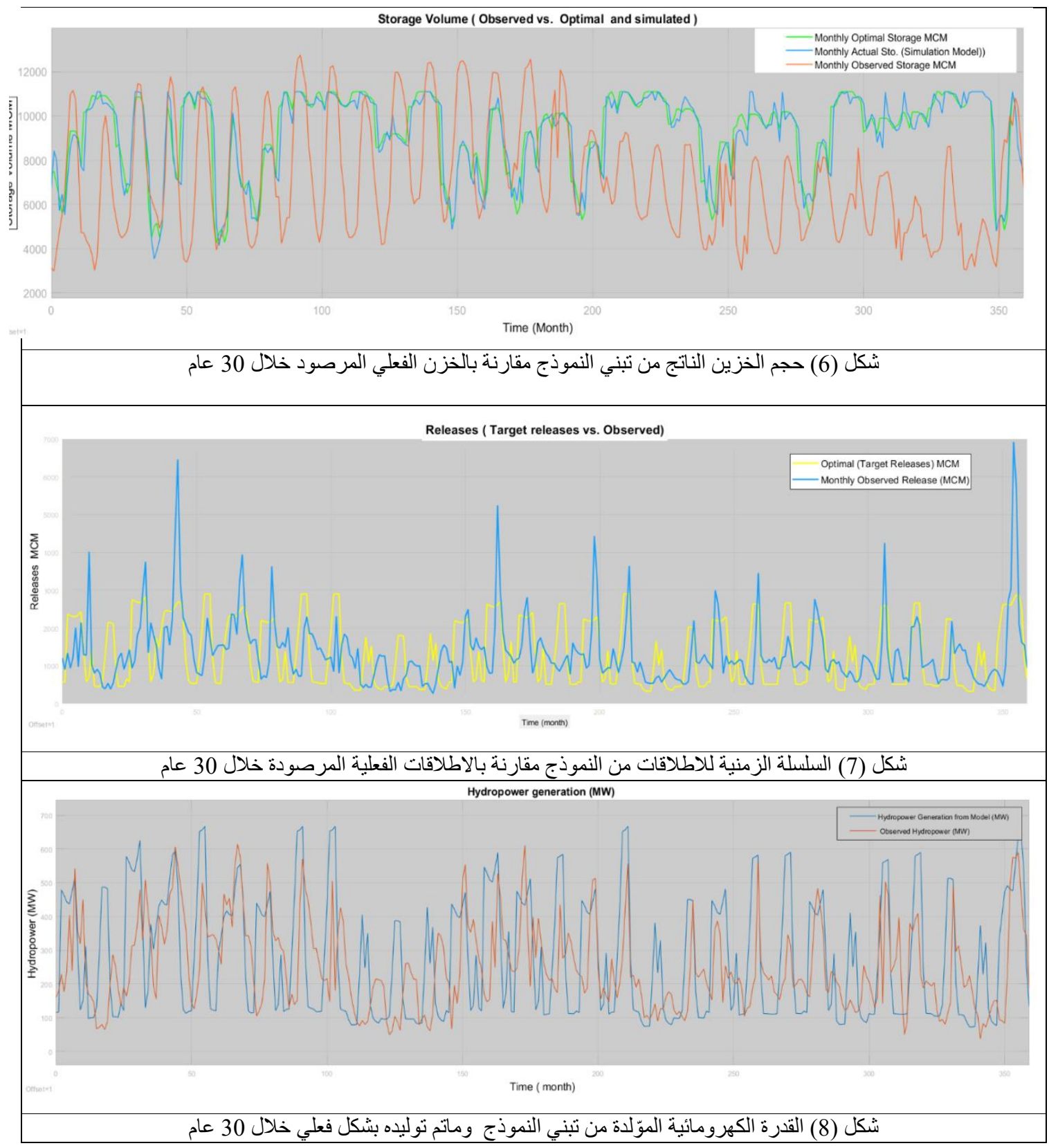

1

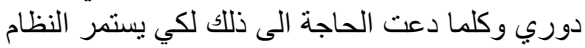

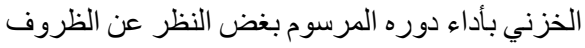

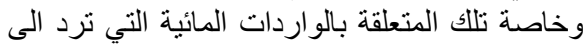
النظام.

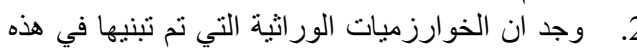

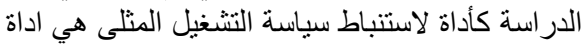

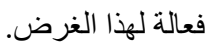

و لأجل التأكد من ان الحل الامثل الذي تم التوصل اليه

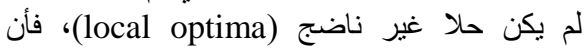
الاستفادة من دو ال الامثلية اللاخطية المقيّة التي تتيحها منصة (Matlab) قد اسفر عن ايجاد حلولا مجدية. ولقد وجد ان عملية التهجين هذه ذات جدوى.

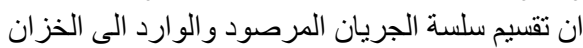
بشكل فئات معلومة المدى يساعد في اعطاء المرونة المرنة .3
يتضح من الثكل اعلاه ان النموذج المقتر ح استطاع توليد ما مقداره

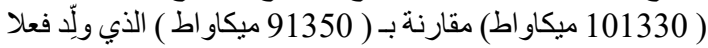

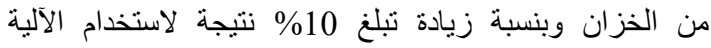

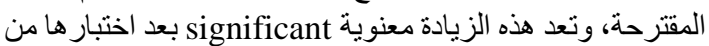

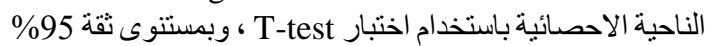

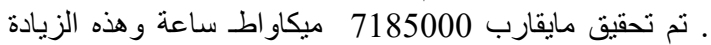

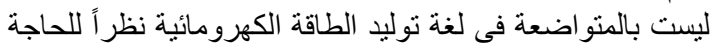
الملحة لكل ميكا واطـ ساعة في ظرف لئ ليد البلد الحالي.

الاستنتاجات و التوصيات تمخضت الدر اسة الحالية عن استتاجات يمكن اجمالها بما يلي: 
genetic and tabu search algorithms. Advances in Civil Engineering, 2018)

[2] S. Santos, Kelly Marina, Alcigeimes B. Celeste, and Ahmed El-Shafie. "ANNs and inflow forecast to aid stochastic optimization of reservoir operation." Journal of Applied Water Engineering and Research 7.4 (2019): 314-323.

[3] B., T. G., and G. Gebresenbet. "Modeling hydropower plant system to improve its reservoir operation." International Journal of Water Resources and Environmental Engineering 4.2 (2010): 87-94.

[4] T., Aida, and Thamer Ahmad Mohammad. "Optimization of cascade hydropower system operation by genetic algorithm to maximize clean energy output." 3.2 (2016): 99-106.

[5] S. Vedula and Mujumdar P.P," Water resources system modiling technique and analysis ' (2005)

[6] N., Nkechi, et al. "A review on applications of evolutionary algorithms to reservoir operation for hydropower production." International Journal of Geological and Environmental Engineering 9.9 (2015): 1153-1159.

[7] A., Jatin, Ashvani Kumar Gosain, and Rakesh Khosa. "Optimisation of multipurpose reservoir operation by coupling soil and water assessment tool (SWAT) and genetic algorithm for optimal operating policy (case study: Ganga River Basin)." Sustainability 10.5 (2018): 1660.

[8] M. Dawood, Kamel A. Al-Mohseen and Shaker A. Jalil "Simulation models for Bekhma Reservoir Operation System (Comparison study) ", Journal of University of Duhok, Vol. 17, No.1 (Pure and Eng. Sciences), Pp 126-136, (2014).

[9] A., Yousif H., et al. "Modelling monthly operation policy for the Mosul Dam, northern Iraq." International Journal of Hydrology Science and Technology 5.2 (2015): 179-193.

[10] O., D. O., et al. "Optimization-based reliability of a multipurpose reservoir by Genetic Algorithms: Jebba Hydropower Dam, Nigeria." Cogent Engineering 5.1 (2018): 1438740.

[11] L. Krishna, K., N. V. UmaMahesh, and A. Srinivasa Prasad. "Optimal multipurpose reservoir operation planning using Genetic Algorithm and Non Linear Programming (GA-NLP) hybrid approach." ISH Journal of Hydraulic Engineering 24.2 (2018): 258-265.

[12] A., Ihsan Fasih Hassan. "Future Prospects for Optimal Operation of Mosul Dam Reservoir", Master Thesis, Department of Water Resources Resources, College of Engineering, University of Mosul. (2018).

[13] A., A. M. Y. Optimal water utilization from Tigris basin reservoirs north of Fatha for hydroelectric power generation. Diss. Ph. D. Thesis, College of Engineering, Water Resources Department, University of Mosul, 2002.

[14] K., Mohammed Awni, and Kamel A. AlMohseen. "Planning and Decision Making Under Uncertainty (Mosul Reservoir Optimal Operating Policy-Case Study)." Al-Rafidain Engineering Journal (AREJ) 25.1 (2020): 85-96.

[15] S., Getting Started Guide, Matlab \& Simulink R2016 a, The MathWorks, Inc. (2016).

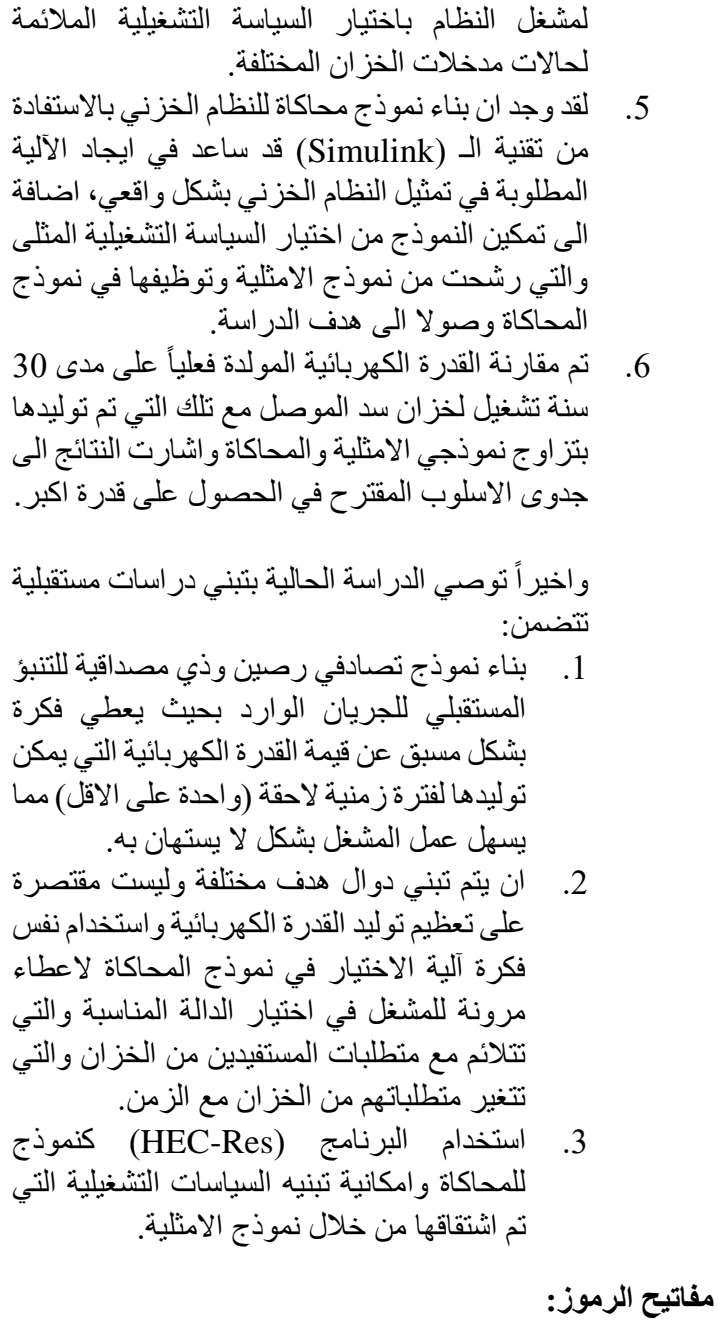

MOP: Mosul operation policy.

GA: Genetic algorithem.

NLP: Non-Linear programming.

GA-NLP: Hybrid approach of Genetic algorithm and nonlinear programming.

$\mathrm{R}$ : Releases from penstock.

$\mathrm{H}$ : Net Head of storage water up stream of power house.

S: Storage volume at end of period $t$.

$\eta$ : Efficiency of the power house plant

I : Inflow

Pr: Precipitation

Ev: Evaporation

L: Losses

fmincon : Minimum of a constrained nonlinear function solver.

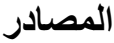

[1] A. Kangrang, , Prasanchum, H., \& Hormwichian, R. (2018). Development of future rule curves for multipurpose reservoir operation using conditional 
[16] K., Steven T. Introduction to Simulink with engineering applications. Orchard Publications, 2006.

[17] MATLAB Optimization-Toolbox User's Guide, The MathWorks, Inc. (2020)

\title{
Appropriate Operating Policy for a Reservoir System Based on Inflow States (Mosul Reservoir as a Case Study)
}

\author{
Ziyad Taher Ali \\ ziyad.enp110@student.uomosul.edu.iq
}

Kamel A. Al-Mohseen

k.almohsee@uomosul.edu.iq

Dams and Water Resources Engineering Department, Collage of Engineering, University of Mosul

\begin{abstract}
:
The present study has focused on the selection of appropriate operating policies for a reservoir system to maximize the hydropower generation through the derivation of operation rule curves for different states of the inflow into the reservoir. And in order to accomplish this task, 30 years of monthly observed inflow has been used and arranged into five intervals with lower and upper values of each interval. The Genetic Algorithms techniques were implemented to obtain the optimal operating policies for each of the proposed five categories of inflow. Furthermore, and in order to alleviate the adverse effects of trapping in local maxima, a hybrid method is suggested making use of the built-in functions of optimization available in the Matlab software. The non-linear optimization function used the values of the variables obtained by GA's as initial solutions required by the optimization process to reach global solutions. One of the main objectives of the current study was to build a simple mechanism to select the most appropriate operating policy which is compatible with the nature of the inflow state entering the reservoir during a certain period of time. Consequently, a simulation model was conducted using SIMULINK technique to mimic the Mosul reservoir system which is selected as a case study to test the proposed method. The results reveal an improvement by 10 $\%$ of hydropower generation over that produced over the 30 years of real operation using the current method.
\end{abstract}

Keywords:

Reservoir operation, Optimization, GAs, Simulation 